\title{
HEREDITARILY CLOSURE-PRESERVING COLLECTIONS AND METRIZATION
}

\author{
D. BURKE, R. ENGELKING AND D. LUTZER ${ }^{1}$
}

ABSTRACT. In this paper we present a generalization of the Nagata-Smirnov metrization theorem. We prove that a regular $T_{1}$-space is metrizable if and only if it has a base of open sets which is the union of countably many hereditarily closure-preserving subcollections. In addition, we investigate intersections of hereditarily closure-preserving collections of open sets.

A collection $K$ of subsets of a space $X$ is closure-preserving if $\operatorname{cl}(\bigcup \mathcal{Q})=\bigcup\{\operatorname{cl}(L) \mid L \in \mathcal{Q}\}$ for any subcollection $\mathcal{Q}$ of $\mathcal{K}$. A collection $\mathcal{H}$ of subsets of $X$ is bereditarily closure-preserving (HCP) if, whenever a subset $K(H) \subset H$ is chosen for each $H \in \mathcal{H}$, the resulting collection $\mathcal{K}=\{K(H) \mid$ $H \in \mathcal{H}\}$ is closure-preserving. Clearly, every locally finite collection is hereditarily closure-preserving. Examples show that closure preserving collections may fail to be HCP and that HCP collections may fail to be locally finite. A $\sigma-H C P$ collection is one which can be written as a countable union of HCP subcollections.

The classical Nagata-Smirnov metrization theorem [4], [5] asserts that a regular space ${ }^{2}$ is metrizable if and only if it has a $\sigma$-locally finite base. Regular spaces which have a $\sigma$-closure-preserving base were introduced by J. Ceder [1]. Ceder called these spaces " $M_{1}$-spaces" and gave examples which show that $M_{1}$-spaces need not be first-countable and that even when they are first-countable they need not be metrizable.

In this paper we consider regular spaces which have a $\sigma$-HCP base. Such spaces appear to lie between metrizable spaces and $M_{1}$-spaces. We will show that they coincide with metrizable spaces.

Lemma 1. Let $X$ be a $T_{1}$-space and suppose $p \in X$ has a neighborhood

Received by the editors August 10, 1973 and, in revised form, June 14, 1974. AMS (MOS) subject classifications (1970). Primary 54E35; Secondary 54D20.

Key words and phrases. Metrizability, Nagata-Smirnov theorem, hereditarily closure-preserving collection.

1 Partially supported by NSF Grant GP 29401.

2 We adopt the convention that regular spaces must be $T_{1}$. Copyright $\odot$ 1975, American Mathematical Society 
base $B$ of cardinality $m$. Let $\mathcal{H}$ be an HCP collection of subsets of $X$ and suppose that no member of $\mathcal{H}$ contains $p$. Then some neighborhood of $p$ meets fewer than $\mathrm{m}$ members of $\mathcal{H}$.

Proof. Let $\Gamma$ be the first ordinal with cardinality $m$. Well order $B$ as $B=\{B(\alpha) \mid \alpha<\Gamma\}$ and suppose each member of $\mathfrak{B}$ meets at least $m$ members of $\mathcal{H}$. Inductively choose members $H(\alpha) \in \mathcal{H}$ for $1 \leq a<\Gamma$ such that

(a) if $a \neq \alpha^{\prime}$ then $H(\alpha) \neq H\left(\alpha^{\prime}\right)$,

(b) $H(\alpha) \cap B(\alpha) \neq \varnothing$.

For each $\alpha<\Gamma$ choose a point $q(\alpha) \in H(\alpha) \cap B(\alpha)$; it is not required that $q(\alpha)$ 's be distinct. Let $K(\alpha)=\{q(\alpha)\}$. Since $\mathcal{H}$ is hereditarily closure preserving the set $K=\bigcup\{K(\alpha) \mid a<\Gamma\}$ must be closed, and $K$ does not contain $p$. Yet each member of $\mathcal{B}$ meets $K$, so $K$ cannot be closed unless it does contain $p$.

Corollary 2. Let $p$ be a point of a $T_{1}$-space $X$. Suppose $p$ has a neighborhood base $\mathcal{B}$ whose cardinality is $m$. If $\mathcal{H}$ is any HCP collection such that for each $H \in \mathcal{H}, p$ is not an isolated point of $H$, then some neighborhood of $p$ meets fewer than $m$ members of $\mathfrak{H}$.

Proof. Let $\mathcal{H}^{\prime}=\{H \backslash\{p\} \mid H \in \mathcal{H}\}$. Lemma 1 yields a neighborhood $B$ of $p$ meeting fewer than $m$ members of $\mathcal{H}^{\prime}$. Since, for a set $H \in \mathcal{H}, B \cap(H \backslash\{p\})$ $\neq \varnothing$ if and only if $B \cap H \neq \varnothing$, we see that $B$ meets fewer than members of $\mathcal{H}$.

Corollary 3. Let $p$ be a nonisolated point of a $T_{1}$-space $X$ and let $\mathcal{H}$ be an HCP collection of open subsets of $X$. If $p$ has a countable neighborhood base, then $\mathcal{H}$ is locally finite at $p$.

Lemma 4. Suppose $p$ is a limit point ${ }^{3}$ of a set $A$ in a space $X$ and that there is a $G_{\delta}$-subset $G$ of $X$ which contains $p$ and has $G \cap(A \backslash\{p\})=$ $\varnothing$. Then any HCP collection of neighborhoods of $p$ must be finite.

Proof. Write $G=\bigcap\{G(n) \mid n \geq 1\}$ where each $G(n)$ is an open subset of $X$. Suppose $\mathcal{C}$ is an infinite HCP collection of neighborhoods of $p$. Let $C(1), C(2), \ldots$ be distinct members of $C$. Define $D(1)=A \cap C(1) \cap G(1)$ and $D(n)=D(n-1) \cap C(n) \cap G(n)$ whenever $n \geq 2$. Because $C(1) \cap G(1)$ is a neighborhood of $p, p$ is a limit point of $D(1) \backslash\{p\}$. However $p$ is not a limit

3 I.e., $p \in \operatorname{cl}(A \backslash\{p\})$. 
point of any set $D(n) \backslash D(n+1)$ so that because the $C(n)$ 's are distinct members of an HCP collection, $p$ cannot be a limit point of the set $\bigcup\{D(n) \backslash D(n+1) \mid n \geq 1\}=D_{1} \backslash\{p\}$. That contradiction establishes the lemma.

Theorem 5. A regular space $X$ is metrizable if and only if $X$ has a $\sigma-H C P$ base of open sets.

Proof. That every metrizable space has such a base follows directly from the Nagata-Smirnov theorem.

To prove the converse assertion, let $B=\bigcup\{B(n) \mid n \geq 1\}$ be a $\sigma$-HCP base for $X$. Let $p$ be a nonisolated point of $X$. Then $\{p\}$ is a $G_{\delta}$-subset of $X$. For each fixed $m$ the collection $\{B \in B(m) \mid p \in B\}$ is finite, in light of Lemma 4 , so that $p$ belongs to only countably many members of $\mathfrak{B}$. Thus $X$ is first-countable at $p$.

Since $X$ is first-countable, it follows from Corollary 3 that each set $X(n)=\{x \in X \mid \Re(n)$ is locally finite at $x\}$ contains all nonisolated points of $X$. Also, each $X(n)$ is an open set. Let $B^{\prime}(n)=\{B \cap X(n) \mid B \in \mathcal{B}(n)\}$. Then each collection $\mathscr{B}^{\prime}(n)$ is locally finite at all points of $X$ and the collection $\mathfrak{B}^{\prime}=\bigcup\left\{\mathfrak{B}^{\prime}(n) \mid n \geq 1\right\}$ contains a neighborhood base at each nonisolated point of $X$.

Let $\mathfrak{B}^{\prime \prime}(n)=\left\{\{x\} \mid\{x\} \in \mathcal{B}_{(n)}\right\}$. Each $\mathcal{B}^{\prime \prime}(n)$ is a discrete collection in $X$ so that the collection $\cup\left\{\Re^{\prime}(n) \cup \Re^{\prime \prime}(n) \mid n \geq 1\right\}$ is a $\sigma$-locally finite base for $X$. According to the Nagata-Smirnov theorem, $X$ is metrizable.

A more subtle application of Lemma 4 yields a result on $\sigma$-HCP local bases at a point.

Theorem 6. Suppose $p$ is a nonisolated point of a $T_{1}$-space $X$ and suppose $\bigcup\{\Re(n) \mid n \geq 1\}$ is a $\sigma-H C P$ base of neighborhoods of $p$. Then each $\mathfrak{B}(n)$ is finite and $X$ is first countable at $p$.

Proof. For each $B \in \mathcal{B}(n)$ choose a point $y(B) \in B \backslash\{p\}$. Since $\mathcal{B}(n)$ is HCP, the set $F(n)=\{y(B) \mid B \in \Re(n)\}$ is a closed set. Furthermore $p$ is a limit point of the set $F=\bigcup\{F(n) \mid n \geq 1\}$. Let $G=X \backslash F$. Then $G$ is a $G_{\delta}$ subset of $X, p \in G$, and $G \cap(F \backslash\{p\})=\varnothing$. According to Lemma 4, each collection $B(n)$ must be finite.

In an attempt to simplify the proofs of Theorems 5 and 6-by eliminating the need for the technical Lemma 4-the authors were led to the conjecture that if $\mathcal{H}$ is an open HCP collection in a space $X$, then $\cap \mathcal{H}$ is open in $X$. Unfortunately, as Example 8 will show, the conjecture is false; however we can prove 
Proposition 7. If $\mathcal{H}$ is an open HCP collection in a Hausdorff $k$-space [3] $X$, then $\cap \mathcal{H}$ is open. ${ }^{4}$

Proof. One shows that for each (countably) compact set $K \subseteq X$, the collection $\{H \cap K \mid H \in \mathcal{H}\}$ contains only finitely many distinct subsets of $K$. Hence $K \cap(\cap \mathcal{H})=\bigcap\{H \cap K \mid H \in \mathcal{H}\}$ is the intersection of finitely many relatively open subsets of $K$, so $K \cap(\cap \mathcal{H}$ ) is relatively open in $K$ for each compact subset $K$ of $X$. Since $X$ is a $k$-space, $\cap \mathcal{H}$ is open in $X$.

Example 8. There is an open HCP collection $\mathcal{H}$ in a hereditarily paracompact space $X$ such that $\cap \mathcal{H}$ is not open.

To construct $X$ we need a definition and a technical lemma. Let us say that a function $f:\left[0, \omega_{1}\left[\rightarrow \mathcal{P}\left(\left[0, \omega_{1}[)^{5}\right.\right.\right.\right.$, where $\omega_{1}$ is the first uncountable ordinal, is admissible if for each $\alpha<\omega_{1}, f(\alpha)$ is a countable subset of $] \alpha, \omega_{1}[$.

Lemma. Let $f:\left[0, \omega_{1}\left[\rightarrow \mathcal{P}\left(\left[0, \omega_{1}[)\right.\right.\right.\right.$ be admissible. Then $\left[1, \omega_{1} \backslash \backslash \bigcup\left\{f(\alpha): 0 \leq \alpha<\omega_{1}\right\}\right.$ is uncountable.

Proof. If $\left[1, \omega_{1} \backslash \backslash \bigcup\left\{f(a): 0 \leq a<\omega_{1}\right\}\right.$ were countable we could obtain an admissible function $g$ such that $\bigcup\left\{g(\alpha) \mid 0 \leq \alpha<\omega_{1}\right\}=\left[\mathrm{r}, \omega_{1}\right.$ [. Defining $\phi(0)=0$ and $\phi(\beta)=\inf \{\alpha: \beta \in g(\alpha)\}$ if $1 \leq \beta<\omega_{1}$, we obtain a function $\phi:\left[0, \omega_{1}\left[\rightarrow\left[0, \omega_{1}\left[\right.\right.\right.\right.$ such that $\phi(\beta)<\beta$ if $0<\beta<\omega_{1}$ and such that $\phi^{-1}(\alpha)$ is countable for each $\alpha<\omega_{1}$. But according to a theorem of Alexandroff and Urysohn [2, p. 79], no such function can exist.

It is clear that if $f$ and $g$ are admissible then so is $h$, defined by $h(\alpha)=f(\alpha) \cup g(\alpha)$ for each $\alpha<\omega_{1}$. For each admissible $f$, let $N_{f}=$ $\left[1, \omega_{1}\right] \backslash \bigcup\left\{f(\alpha): 0 \leq \alpha<\omega_{1}\right\}$ and let $\pi=\left\{N_{f}: f\right.$ is admissible $\}$. Then $\pi$ is closed under finite intersections so that we may topologize the set $X=$ $\left[0, \omega_{1}\right]$ by taking $\pi$ to be a neighborhood base at $\omega_{1}$ and by making each other point isolated. With that topology $X$ is a hereditarily paracompact Hausdorff space.

4 One can also prove that if $\mathcal{H}$ is an open HCP collection in a locally connected regular space then $\bigcap_{\mathcal{H}}$ is open, the key lemma being that in any $T_{1}$-space the intersection of an open, countable, HCP collection is open. Given that lemma, suppose $x \in \bigcap_{H}$ where $H$ is an open HCP collection in $X$. If $H$ were infinite we could choose distinct members $H_{n}$ of $\boldsymbol{H}$ and (using regularity and local connectedness of $X$ ) connected open sets $U_{n} \subset H_{n}$ in such a way that $x \in U_{n}$ and $\operatorname{cl}\left(U_{n+1}\right)$ $\subsetneq U_{n} \cap H_{n+1}$ for each $n$. But then the set $\bigcap\left\{U_{n}: n \geq 1\right\}$ would be a nonempty, closed-and-open subset of $X$ which is properly contained in the connected set $U_{1}$. That being impossible, $\boldsymbol{H}$ is finite and our assertion is established.

$5 \rho\left(\left[0, \omega_{1}[)\right.\right.$ denotes the power set of $\left[0, \omega_{1}[\right.$. 
For each $\alpha<\omega_{1}$, let $\left.\left.H_{a}=\right] \alpha, \omega_{1}\right]$ and let $\mathcal{H}=\left\{H_{\alpha}: 0 \leq a<\omega_{1}\right\}$. Then $\mathcal{H}$ is a collection of open sets in $X$ and $\cap \mathcal{H}=\left\{\omega_{1}\right\}$ is not open. To complete the example, we show that $\mathcal{H}$ is HCP. Suppose that $S_{\alpha} \subseteq H_{a}$ for each $\alpha<$ $\omega_{1}$; it will be enough to show that if $\omega_{1} \notin \operatorname{cl}\left(S_{a}\right)$ for each $\alpha$, then $\omega_{1} \notin$ $\operatorname{cl}\left(\bigcup\left\{S_{a}: \alpha<\omega_{1}\right\}\right)$. Because $\omega_{1} \notin \operatorname{cl}\left(S_{\alpha}\right)$ there is an admissible function $f_{a}$ having $S_{a} \subseteq \bigcup\left\{f_{a}(\beta): 0 \leq \beta<\omega_{1}\right\}$. By modifying $f_{a}$ if necessary, we may assume that $f_{\alpha}(\beta)=\varnothing$ whenever $\beta<\alpha$. Defining $g(\beta)=\bigcup\left\{f_{\alpha}(\beta): 0 \leq \alpha \leq\right.$ $\beta\}$ for $\beta<\omega_{1}$, we obtain an admissible function having $\bigcup\left\{S_{a}: 0 \leq \alpha<\omega_{1}\right\}$ $\subseteq \bigcup\left\{g(\beta): 0 \leq \beta<\omega_{1}\right\}$ so that $\omega_{1} \notin \operatorname{cl}\left(\bigcup\left\{S_{a}: 0 \leq a<\omega_{1}\right\}\right)$ as required.

The referee has suggested a possible improvement in our metrization theorem. Suppose that one considers collections $\mathcal{H}$ in a space $X$ with the property that if a point $x(H) \in H$ is chosen for each $H \in \mathcal{H}$ then the set $\{x(H): H \in \mathcal{H}\}$ is a closed discrete subspace of $X$; such collections might reasonably be called weakly $H C P$. Then is it true that a regular space is metrizable if it has a $\sigma$-weakly HCP base? The question has an affirmative answer provided only $k$-spaces are considered: the proof of Proposition 7 shows that a $k$-space having a $\sigma$-weakly HCP base must be first countable so that the proof of Theorem 5, beginning with the third paragraph, shows that $X$ (if regular) is metrizable. However, our next example shows that if $X$ is not assumed to be a $k$-space, then the suggested generalization of Theorem 5 is false.

Example 9. There is a nonmetrizable, hereditarily paracompact space which has a $\sigma$-weakly HCP base.

Proof. Let $A$ be the set of all ordinals having cardinality less than $\boldsymbol{\kappa}_{\omega_{0}}$. Let $Z$ be the product space $\{0,1\}^{A}$ and let $\overline{0}$ be the element of $Z$ having $\overline{0}(\alpha)=0$ for each $\alpha \in A$. Let $X$ be the set $\{\overline{0}\} \cup\{z \in Z$ : the set $\{a \in A: z(\alpha)=0\}$ is finite $\}$. Topologize $X$ by making each point of $X \backslash\{\overline{0}\}$ isolated and by taking basic neighborhoods of $\overline{0}$ to be all sets of the form $U \cap X$ where $U$ is a basic neighborhood of $\overline{0}$ in the product space $Z$. Then $X$ is hereditarily paracompact and nonmetrizable.

Let $\mathfrak{B}^{\prime}(n)=\{\{z\}: z \in X \backslash\{\overline{0}\}$ and $|\{\alpha \in A: z(\alpha)=0\}|=n\}$. Then each $\mathfrak{B}^{\prime}(n)$ is a discrete collection in $X$. For each basic open neighborhood $U$ of $\overline{0}$ in $Z$ the set $R(U)=\left\{a \in A: \pi_{a}[U]=\{0\}\right\}$ is finite, where $\pi_{a}: Z \rightarrow$ $\{0,1\}_{a}$ denotes the projection. Let $\mathfrak{B}^{\prime \prime}(n)=\{U \cap X: U$ is a basic neighborhood of $\overline{0}$ in $Z$ and $R(U) \subseteq\left[0, \omega_{n}[\}\right.$, where $\omega_{n}$ is the first ordinal of cardinality $\aleph_{n}$. Since each set $U \cap X^{n}$ in $\mathfrak{B}^{\prime \prime}(n)$ is uniquely determined by the finite set $R(U)$ of $\left[0, \omega_{n}\left[,\left|\mathcal{B}^{\prime \prime}(n)\right| \leq \aleph_{n}\right.\right.$. In order to show that $\mathcal{B}^{\prime \prime}(n)$ is weak- 
ly HCP it will be sufficient to show that if a point $z_{U} \in(U \cap X) \backslash\{\overline{0}\}$ is chosen for each $U \cap X \in \mathscr{B}^{\prime \prime}(n)$, then $\overline{0} \notin \operatorname{cl}\left\{z_{U}: U \cap X \in \mathfrak{B}^{\prime \prime}(n)\right\}$. For each of the chosen points $z_{U}$, the set $S(U)=\left\{\alpha \in A: z_{U}(\alpha)=0\right\}$ is finite. Since $\left|B^{\prime \prime}(n)\right| \leq \boldsymbol{X}_{n}$ the set $S=\bigcup\left\{S(U): U \cap X \in B^{\prime \prime}(n)\right\}$ has cardinality not exceeding $\boldsymbol{K}_{n}$ so that we may choose $\beta \in A \backslash S$. But then the neighborhood $X \cap$ $\{z \in Z: z(\beta)=0\}$ of $\overline{0}$ contains no point $z_{U}$ so that $\overline{0} \notin \operatorname{cl}\left\{z_{U}: U \cap X \epsilon\right.$ $\left.\mathfrak{B}^{\prime \prime}(n)\right\}$, as required.

Since the collection $\bigcup\left\{\mathcal{B}^{\prime}(n) \cup \mathcal{B}^{\prime \prime}(n): n \geq 1\right\}$ is a base for $X$, the proof is complete.

\section{BIBLIOGRAPHY}

1. J. Ceder, Some generalization of metric spaces, Pacific J. Math. 11 (1961), 105-125. MR 24 \#A 1707.

2. I. Juhász, Cardinal functions in topology, Math. Center Tracts, North-Holland, Amsterdam, 1971.

3. J. Kelley, General topology, Van Nostrand, Princeton, N. J., 1955. MR 16, 1136.

4. J. Nagata, On a necessary and sufficient condition of metrizability, J. Inst. Polytech. Osaka City Univ. Ser A Math. 1 (1950), 93-100. MR 13, 264.

5. Ju. M. Smirnov, A necessary and sufficient condition for metrizability of a topological space, Dokl. Akad. Nauk SSSR 77 (1951), 197-200. (Russian) MR 12, 845.

DEPARTMENT OF MATHEMATICS, MIAMI UNIVERSITY, OXFORD, OHIO 45056

DEPARTMENT OF MATHEMATICS, UNIVERSITY OF PITTSBURGH, PITTSBURGH, PENNSYLVANIA 15260 